\title{
A New Excitation Technique for Wideband Short Backfire Antennas
}

RongLin Li, Dane Thompson, John Papapolymerou, Joy Laskar, and Manos M. Tentzeris

\author{
Georgia Electronic Design Center \\ School of Electrical and Computer Engineering \\ Georgia Institute of Technology \\ Atlanta, GA 30332-0250, U.S.A. \\ E-mail: rlli@ece.gatech.edu \\ Phone: 404-385-6003 \\ Fax: $\quad 404-894-0222$
}

\begin{abstract}
A new excitation technique is developed to improve the impedance bandwidth and to lower the manufacturing cost of a short backfire antenna (SBA). The new excitation structure consists of a planar monopole and a microstrip feed line, both of which are printed on the same dielectric substrate. By splitting the printed monopole with a slot, a wideband performance can be achieved. It is demonstrated that the splitmonopole-excited SBA can achieve an impedance bandwidth of about 15\% (VSWR<2) while maintaining the good radiation performance. As an example, an SBA configuration with the new excitation topology designed at the $5 \mathrm{GHz}$ UNII band is described and the simulation and experimental results show good agreement. The effects of the geometric parameters of the excitation structure on the impedance performance are investigated and the operating mechanism of the split-monopoleexcited SBA is discussed. Being a low-cost, high-gain, and wideband directional antenna, the new SBA can find applications in various wireless systems, such as LMDS, WLAN, and the emerging WiMAX networks.
\end{abstract}

Index terms - Excitation technique, short backfire antenna, wideband antenna, lowcost antenna, wireless applications. 


\section{Introduction}

In recent years, there is an increasing need for high-gain wideband directional antennas in wireless applications [1], such as the local multipoint distribution service (LMDS) systems [2] and the millimeter-wave wireless local area networks (WLAN) [3]. In particular, WiMax (world interoperability for microwave access), a technology based on an evolving standard for broadband point-to-multipoint wireless networking, is becoming a hot spot in wireless industry [4], [5]. For a WiMax system, it is typical to use fixed, externally mounted (usually on rooftops or external walls) directional subscriber antennas to communicate with base stations which are connected to the Internet. Since one of the major goals for wireless systems is to offer a less expensive infrastructure than a wired one (such as that based on a T1, DSL or cable connection), the cost-effectiveness of a wireless deployment is a big issue of concern. Also for the reasons of system flexibility and interoperability, the ability for a wireless antenna to operate at a wide frequency band, covering more than one standard, is highly desirable.

The short backfire antenna (SBA), developed first in the 1960s [6], [7], may become one of the most competitive candidates for these wireless application because of its low profile, high gain, lightweight, and high isolation from surroundings if its impedance bandwidth can be improved and its manufacturing cost can be further reduced. The SBA has been widely used in mobile/maritime satellite communications, tracking, and telemetry [8], due to its excellent radiation characteristics (a gain on the order of 13-15 dBi, with sidelobes of at least $-20 \mathrm{~dB}$ and a backlobe lower than $-30 \mathrm{~dB}$ ) [9], [10], its compact structure $\left(\sim 0.5 \lambda_{0}\right.$ in height, $\lambda_{0}$ is the free-space wavelength at operating frequency), and its simple feed configuration (a single dipole rather than an array). The most popular excitation for the SBA is a half-wave dipole antenna, which has a simple structure. Unfortunately the dipole-excited SBA has a narrow frequency 
bandwidth for its input impedance since the SBA is essentially a leaky cavity structure. The natural (i.e. without matching circuit) impedance match bandwidth for a crossdipole excited (for circularly polarization) SBA is only 3-5 \% for the voltage standing wave ratio (VSWR) under 1.5 [11], [12]. This situation becomes even worse for a single-dipole excited (for linear polarization) SBA. The authors' investigation shows that the bandwidth of a single-dipole excited SBA is less than $1 \%$ for $\operatorname{VSWR}<2$ if no matching circuit is involved.

An alternative excitation configuration that may improve the impedance bandwidth is the waveguide feed system, such as the coaxial or rectangular waveguide feed [13], [14], which has a bulky structure and requires complicated manufacturing process. Recently, the authors have developed an unbalance-fed slot-excited SBA to enhance the impedance bandwidth [15]. It has been demonstrated that the slot-excited SBA can achieve a bandwidth for VSWR $<2$ of more than $20 \%$. However, a pair of wire pins is needed to support the excitation slot, which increases the complexity for fabrication and thus raises the manufacturing cost. This is critical, especially for millimeter-wave applications, because thinner (sometimes too thin to process mechanically) wires are required for the higher frequency bands (e.g. the wire diameter may be less than $0.2 \mathrm{~mm}$ in $60 \mathrm{GHz}$ band).

In this paper, a new excitation technique is proposed for simplifying the excitation structure and maintaining the wideband impedance performance. The proposed excitation configuration consists of a planar monopole fed by a microstrip line. Both the monopole and the microstrip feed line are printed on a dielectric substrate or a printed circuit board (PCB). By splitting the printed monopole with a slot, the wideband performance can be achieved. Because the excitation structure is entirely fabricated on the same PCB, the manufacturing cost for the wideband SBA can be 
significantly reduced by taking advantage of any modern PCB technology and with the help of high-volume production.

In the next section, the geometry of the new excitation structure for the SBA is described. Simulation and experimental results for impedance performance and radiation patterns are then presented. Finally the effects of its geometric parameters on the impedance performance are investigated and the operating mechanism of the new SBA is discussed.

\section{The New Excitation Topology}

Consider an SBA that is excited by the new excitation structure, as depicted in Fig. 1. The SBA contains a primary reflector (diameter $=\mathrm{D}_{\mathrm{r}}$ ), a sub-reflector (diameter $=\mathrm{D}_{\mathrm{s}}$ ), and a circular rim. The sub-reflector is printed on a thin dielectric substrate (thickness=t). The height $\left(\mathrm{H}_{\mathrm{r}}\right)$ of the rim is chosen to be almost the same of the height $\left(\mathrm{H}_{\mathrm{s}}=\mathrm{t}+\mathrm{H}_{\mathrm{r}}\right)$ of the sub-reflector based on the following considerations: (a) to minimize the backside radiation, (b) to increase the isolation from surroundings so that the antenna may be embedded in the wall of a building, and (c) to support the sub-reflector. The whole structure of the SBA looks like a "can" and the dielectric substrate with the subreflector serves as the cover (or radome) of the "can” (or the SBA). The new excitation structure consists of a planar monopole and a microstrip feed line, both of which are printed on a dielectric substrate. To achieve a wideband performance for input impedance, the printed monopole is split along its central line into two parts with a slot that consists of two rectangles: a narrower one on the upper part and a wider one on the lower part of the monopole. The split monopole is mounted at the center of the primary reflector and is fed across the wider part of the splitting slot by a feed probe which is formed through an extension of a $50-\Omega$ microstrip line. The microstrip feed line is 
printed on the same dielectric substrate but on the opposite side with respect to the split monopole (thus there is no direct electrical connection between the monopole and the feed probe). This type of feeding arrangement has been widely used for broadband printed dipoles [16]-[18]. Note that the authors have tried initially to use the microstripfed dipole as an excitation for the SBA, but it failed in the improvement of impedance bandwidth. A semi-miniaturized type-A (SMA) connector is connected to the microstrip feed line from the backside of the primary reflector. A “close-up” view of the new excitation topology is illustrated in Fig. 2.

Based on the configuration described above, a split-monopole-excited SBA is designed at a center frequency of $f_{c}=5.5 \mathrm{GHz}$ to cover the $5 \mathrm{GHz}$ Unlicensed National Information Infrastructure band (UNII) which include the frequency segments 5.155.35 GHz and 5.725-5.85 GHz. Both the sub-reflector and the excitation structure are etched on an RT/duroid 5880 substrate $\left(\varepsilon_{\mathrm{r}}=2.2, \mathrm{t}=0.508 \mathrm{~mm}\right.$, loss tangent $\left.=0.0009\right)$. The diameters of the primary reflector (or the rim) and the sub-reflector, and the height of the sub-reflector (or the rim) are determined for a desirable radiation performance, such as a higher gain and a lower sidelobe level [19], [20], while other geometric parameters in the excitation topology are optimized for a good impedance performance, i.e. a wide bandwidth for input impedance. Following a large number of numerical full-wave simulations using Microstripes 6.0 (a software based on the Transmission-Line-Matrix technique), all the optimized geometric parameters are obtained and listed in Table I. The overall dimensions of optimized SBA are $0.64 \lambda_{0}$ in height and $2.2 \lambda_{0}$ in diameter. The height of the split-monopole-excited SBA is $0.14 \lambda_{0}$ larger than the typical height $\left(0.5 \lambda_{0}\right)$ for a dipole-excited SBA because of the height occupied by the excitation monopole $\left(0.37 \lambda_{0}\right)$. Note that the height of the excitation dipole is typically $0.25 \lambda_{0}$ [9]. 


\section{Results}

Fig. 3 shows the simulated and measured results of VSWR for the split-monopoleexcited SBA and good agreement is observed. The VSWR $<2$ bandwidth (about 15\%) covers entirely the $5 \mathrm{GHz}$ USII band. The comparison between the measured E-plane, H-plane, and $\phi=45^{\circ}$ plane patterns and the simulations is shown in Fig. 4 at the frequencies of 5.3 and $5.7 \mathrm{GHz}$. The agreement between the measured and simulated results is good over the main beam region. The sidelobe level is lower than $-15 \mathrm{~dB}$ while the peak backlobe is smaller than $-25 \mathrm{~dB}$. The higher sidelobe level is mainly due to the spurious radiation from the microstrip feed line while the very low backlobe level benefits from the increased rim height. Similar to the dipole-excited SBA, the split-monopole-excited SBA has a peak cross-polarized component in the $\phi=45^{\circ}$ plane with a value of around $-15 \mathrm{~dB}$, which is slightly higher than the $-20 \mathrm{~dB}$ for the dipoleexcited SBA due to the higher rim height [20]. Note that the peak of the cross polarization appears beyond the half-power main beam region. The higher measured cross-polarization level than the simulation result is probably due to a slight polarization mismatch occurred during the measurement, e.g., a misalignment between the transmitting antenna (a standard antenna) and the receiving antenna (the antenna under test).

The simulated directivity and measured power gain are plotted in Fig. 5 as a function of frequency. It can be seen that the power gain, slightly lower than the directivity, keeps near $15 \mathrm{dBi}$ over the $5 \mathrm{GHz}$ USII bands. The simulation reveals that the radiation efficiency of the split-monopole-excited SBA (a conductivity of $1.6 \times 10^{7}$ $\mathrm{S} / \mathrm{m}$ was used in the simulation for the whole antenna structure) is higher than $95 \%$ over the frequency range of 5.1-5.9 GHz. The simulated and measured results for the half-power beam width (HPBW) also agree well (see Fig. 5). In the frequency band 
5.1-5.9 GHz, the HPBW in the E-plane maintains almost a constant of $35^{\circ}$ while the HPBW in H-plane decreases as the frequency increases. At the center frequency (5.5 $\mathrm{GHz}$ ) the HPBW in the E-plane is about $10^{\circ}$ wider than that in the H-plane. This phenomenon is similar to that observed for a circular patch antenna where the beamwidth in the E-plane is usually larger than that in the H-plane due to the effect of ground plane [21]. The aperture efficiency at the center frequency is about $\eta_{a}=70 \%$, about $10 \%$ lower than that of a dipole-excited SBA [10]. This explains why a slightly larger antenna aperture (i.e. $\mathrm{D}_{\mathrm{r}}=2.2 \lambda_{0}$ ) is needed for a gain (about $15 \mathrm{dBi}$ ) that the dipole-excited SBA can achieve with an aperture diameter of $2.0 \lambda_{0}[20]$.

\section{Parametric Study and Discussion}

To understand the operating principle of the new excitation topology, a parametric study of the input impedance is performed. For the split-monopole-excited SBA, the input impedance is affected mainly by the sub-reflector and the excitation structure which include the split monopole and the feed probe. (In the parametric study, each time only one parameter varies near its optimized value while all other parameters are fixed at their optimized values.)

First let us check the effect of the sub-reflector on the impedance performance. Fig. 6 shows the impedance locus variation (plotted on a Smith chart) as the sub-reflector moves into the excitation structure. Note that the reference plane for the input impedance is chosen at the interface between the feed probe and the microstrip feed line. From this figure, a poor impedance performance is observed if there is no subreflector. When the sub-reflector is introduced (e. g. $\mathrm{H}_{\mathrm{s}}=40 \mathrm{~mm}$ ), an impedance loop is produced due to its coupling with the excitation structure. As the sub-reflector moves 
toward the excitation structure (that is, its height $\mathrm{H}_{\mathrm{s}}$ reduces), the impedance loop becomes broader because of a stronger coupling. An optimized impedance performance is obtained when $\mathrm{H}_{\mathrm{s}}=35 \mathrm{~mm}$. If the sub-reflector further moves into the excitation structure (e.g. $\mathrm{H}_{\mathrm{s}}=33 \mathrm{~mm}$ ), the impedance loop expands beyond the SWR=2 circle as a result of the over-coupling.

The most direct effect on the impedance performance comes from the feed probe. Fig. 7 shows the impedance variations with its position and dimensions. Fig. 7(a) indicates that the impedance locus moves clockwise as the height of the feed probe $\left(h_{p}\right)$ decreases. This is because a decrease in $h_{p}$ corresponds to moving away from a load (i.e. the radiation resistance). Fig. $7(\mathrm{~b})$ indicates that as the length of the feed probe $\left(l_{p}\right)$ increases, the impedance locus moves toward the upper half of the Smith chart. This is due to the increased inductance caused by the extended feed probe $\left(l_{\mathrm{p}} \uparrow\right)$. Fig. 7 (c) exhibits a slight effect from the width of the feed probe $\left(w_{p}\right)$. This is reasonable since both the coupling capacitance between the feed probe and the monopole and the inductance of the feed probe decrease with $w_{p}$.

Another important factor that affects the impedance performance is the splitting slot. Fig. 8(a) shows the effect of the width of upper rectangular slot $\left(w_{u}\right)$. It can be seen that this narrow slot is essential for the impedance match. Without the slot (i.e. $w_{u}=0$ ), the impedance loop moves far away from the center of the Smith chart. Also it is noticed that the slot cannot be made too wide because as $w_{u}$ increases the impedance loop becomes broader and more capacitive. Fig. 8(b) shows the variation of the impedance locus as the length of the upper rectangular slot $\left(l_{u}\right)$ increases. This tendency is similar to that shown in Fig. 7(a). This result is not difficult to understand because an increase in $\left(l_{u}\right)$ corresponds to a decrease in the length $\left(\mathrm{L}_{\mathrm{m}}-h_{p}-l_{u}\right)$ of the slot line between the feed probe and the narrow slot. The effect of the width of the lower slot 
line $\left(w_{l}\right)$ is plotted in Fig. 8(c). The variation tendency is similar to that shown in Fig. 7(b) for $l_{\mathrm{p}}$. This is obvious since widening $w_{l}$ is equivalent to lengthening $l_{\mathrm{p}}$.

Finally let us examine the effect of the size of the split monopole on the impedance performance. Fig. 9(a) shows a variation tendency similar to that shown in Fig. 7(a). The explanation for this result is straightforward: an increase in the length of the excitation monopole $\left(\mathrm{L}_{\mathrm{m}}\right)$ directly leads to an increase in the length of the upper slot line ( $\left.\mathrm{L}_{\mathrm{m}}-h_{p}-l_{u}\right)$ (note that $h_{p}$ and $l_{u}$ are fixed as $\mathrm{L}_{\mathrm{m}}$ increases). Fig. 9(b) shows the effect of the width of the planar monopole $\left(\mathrm{W}_{m}\right)$. It is seen that the impedance loop becomes broader as $\mathrm{W}_{m}$ increases because of the increased coupling between the widened excitation monopole and the sub-reflector.

To further understand the operating mechanism of the split-monopole-excited SBA, the field distributions around the excitation structure are simulated. It is found that the current distribution on the split monopole is concentrated on the upper part of the splitting slot. It is this current distribution that causes a strong electric (or capacitive) coupling between the excitation monopole and the sub-reflector. It is also observed that the magnetic field lines are closed up around the sub-reflector and the feed probe, respectively. This confirms that there is no magnetic linkage between the sub-reflector and the excitation monopole. Due to the electric coupling between the sub-reflector and the excitation monopole, a strong fringing field distribution is produced at the edge of the sub-reflector. The fringing field is the major contributor to the radiation field of the SBA. The simulated field distribution also demonstrates the effect of the rim which effectively constrains the electromagnetic field within the aperture formed by the rim and the sub-reflector. It is found that the field outside the aperture is more $40 \mathrm{~dB}$ lower than the fringing field. The constrained field distribution contributes to two benefits: 1) 
to increase the effective area of the aperture, thus enhancing the directivity of the SBA, and 2) to reduce the interference between the SBA and its surroundings.

\section{Conclusion}

Mainly motivated by the emerging WiMax technology where a larger number of lowcost high-gain directional subscriber antennas are needed, a novel cost-effective excitation technique has been developed for wideband short backfire antennas (SBAs). The excitation structure proposed consists of a planar monopole and a microstrip feed line, both of which are printed on the same dielectric substrate, thus helpful in lowering manufacturing cost. By introducing a slot into the printed monopole, the impedance bandwidth of the new SBA can be increased from less than $1 \%$ for the typical dipoleexcited SBA to about $15 \%$ for VSWR $<2$. Over this bandwidth, the SBA has a gain of near $15 \mathrm{dBi}$, a backlobe level of less than $-25 \mathrm{~dB}$, and sidelobes lower than $-15 \mathrm{~dB}$. The

new excitation technique implemented in the $5 \mathrm{GHz}$ USII band can be extended to millimeter-wave bands and may find applications in other broadband wireless systems, such as the 27-31 GHz LMDS and the 59-64 GHz short-range broadband WLAN's.

\section{Acknowledgement}

The authors wish to acknowledge the support of Georgia Electronic Design Center (GEDC), the NSF CAREER Award ECS-9984761, the NSF Grant ECS-0313951 and the NSF Packaging Research Center. The authors would like to thank the Reviewers for their valuable suggestions. 


\section{References}

[1] P. F. Driessen, "Gigabit/s indoor wireless systems with directional antennas," IEEE Trans. On Communications, vol. 44, no. 8, pp. 1034-1044, Aug. 1996.

[2] R. B. Waterhouse, D. Novak, A. Nirmalathas, and C. Lim, "Broadband printed sectored coverage antennas for millimeter-wave wireless applications,” IEEE Trans. Antenna and Propagat., vol. 50, no. 1, pp. 12-16, Jan. 2002.

[3] S. Yang, S. H. Tan, and J. S. Fu, "Short backfire antennas for wireless LAN applications at millimeter-waves,” IEEE AP-S International Symposium, vol. 3, pp. 1260-1263, July 2000.

[4] S. J. Vaughan-Nichols, “Achieving wireless broadband with MiMax,” Computer, pp. 10-13, June 2004.

[5] S. M. Cherry, "WiMax and Wi-Fi: separated and unequal," IEEE Spectrum, p. 19, March 2003.

[6] H. W. Ehrenspeck, "The backfire antenna, a new type of directional line source," Proc. IRE, vol. 48, no. 1, pp. 109-110, Jan. 1960.

[7] H. W. Ehrenspeck, “The short-backfire antenna,” Proc. IEEE. vol. 53, no. 6, pp. 11381140, Aug. 1965.

[8] Y. Yamada, T. Takan, and N. Ishida, “Compact antenna equipment for maritime satellite communication systems,” Trans. IECE, vol. 62-B, pp. 844-846, 1979.

[9] K. M. Chen, D. P. Nyquist, J. L. Lin, "Radiation fields of the short-backfire antenna," IEEE Trans. Antenna and Propagat., vol. 16, no. 5, pp. 596-597, Sept. 1968.

[10] K. Fujimoto and J. R. James (Editors), Mobile antenna systems handbook, Second Edition, Norwood, MA: Artech House, pp. 542-545, 2000.

[11] A. Kumar and H. D. Hristov, Microwave Cavity Antennas, Norwood, MA: Artech House, 1989. 
[12] S. Ohmori, S. Miura, K. Kameyama, and H. Yoshimura, "An improvement in electrical characteristics of a short backfire antenna," IEEE Trans. Antenna and Propagat., vol. 31, no. 4, pp. 644-646, July 1983.

[13] A. A. Kishk and L. Shafai, "Gain optimization of short-backfire antenna with different excitation types,” IEEE AP-S International Symposium, vol. 24, pp. 923-926, June 1986.

[14] M. S. Leong, P. S. Kooi, Chandra, and T. S. Yeo, "Theoretical and experimental investigations of two-dimensional waveguide-excited short backfire antenna structure,” IEE Proceedings-H, vol. 136, no. 3, June 1989.

[15] R. L. Li, D. Thompson, M. M. Tentzeris, J. Laskar, "Development of a Wideband Short Backfire Antenna Excited by an Unbalance-Fed H-Shaped Slot,” to be published in IEEE Trans. Antenna and Propagat., Feb. 2005.

[16] B. Edward and D. Rees, “A broadband printed dipole with integrated balun," Microwave. J., pp. 339-344. May 1987.

[17] K. M. K. H. Leong, Y. Qing, and T. Itoh, “Surface wave enhanced broadband planar antenna for wireless applications,” IEEE Microwave and wireless components letters, vol. 11, no. 2, pp.62-64, Feb. 2001.

[18] C. Sabatier, "T-dipole arrays for mobile applications," IEEE Antenna and Propagation Magazine, vol. 45, no. 6, pp. 9-26, Dec. 2003.

[19] G. P. Otto, C. LU, and W. C. Chew, “Circular short backfire antenna modeling,” IEEE Trans. Antenna and Propagat., vol. 40, no. 11, pp. 1434-1438, Nov. 1992.

[20] M. Rayner, A. D. Olver, A. D. Monk, “FD-TD design of short backfire antennas,” IEE Proc.-Microwave. Antennas Propag., vol. 144, no. 1, pp.1-6, Feb. 1997.

[21] R. Garg, P. Bhartia, I. Bahl, and A. Ittipiboon, Microstrip antenna design handbook, Norwood, MA, Artech House, pp. 364-366, 2001. 


\section{List of Captions}

Fig. 1. Short backfire antenna excited by a new excitation structure which consists of a split monopole and a microstrip feed line (the dielectric cover on which the subreflector is printed is hidden).

Fig. 2. A “close-up” view of the new excitation topology.

Table I. Optimized geometric parameters for the split-monopole-excited SBA ( $\lambda_{0}=$ the free-space wavelength at the center frequency)

Fig. 3. Measured and simulated results of VSWR for the split-monopole-excited SBA (inset are two pictures of the antenna prototype).

Fig. 4. Comparison of measured and simulated radiation patterns.
(a) At $5.3 \mathrm{GHz}$
(b) At $5.7 \mathrm{GHz}$

Fig. 5. Directivity, gain, and half-power beam width (HPBW) as a function of frequency.

Fig. 6. Effect of the sub-reflector on the impedance performance.

Fig. 7. Effect of the feed probe on the impedance performance.
(a) Effect of the height of the feed probe $\left(h_{p}\right)$
(b) Effect of the length of the feed probe $\left(l_{p}\right)$
(c) Effect of the width of the feed probe $\left(\mathrm{w}_{\mathrm{p}}\right)$

Fig. 8. Effect of the splitting slot on the impedance performance.
(a) Effect of the width of the upper rectangular slot $\left(w_{\mathrm{u}}\right)$
(b) Effect of the length of the upper rectangular slot $\left(l_{u}\right)$
(c) Effect of the width of the lower rectangular slot $\left(w_{l}\right)$

Fig. 9. Effect of the split monopole on the impedance performance.

(a) Effect of the length of the split monopole $\left(\mathrm{L}_{\mathrm{m}}\right)$

(b) Effect of the width of the split monopole $\left(\mathrm{W}_{\mathrm{m}}\right)$ 


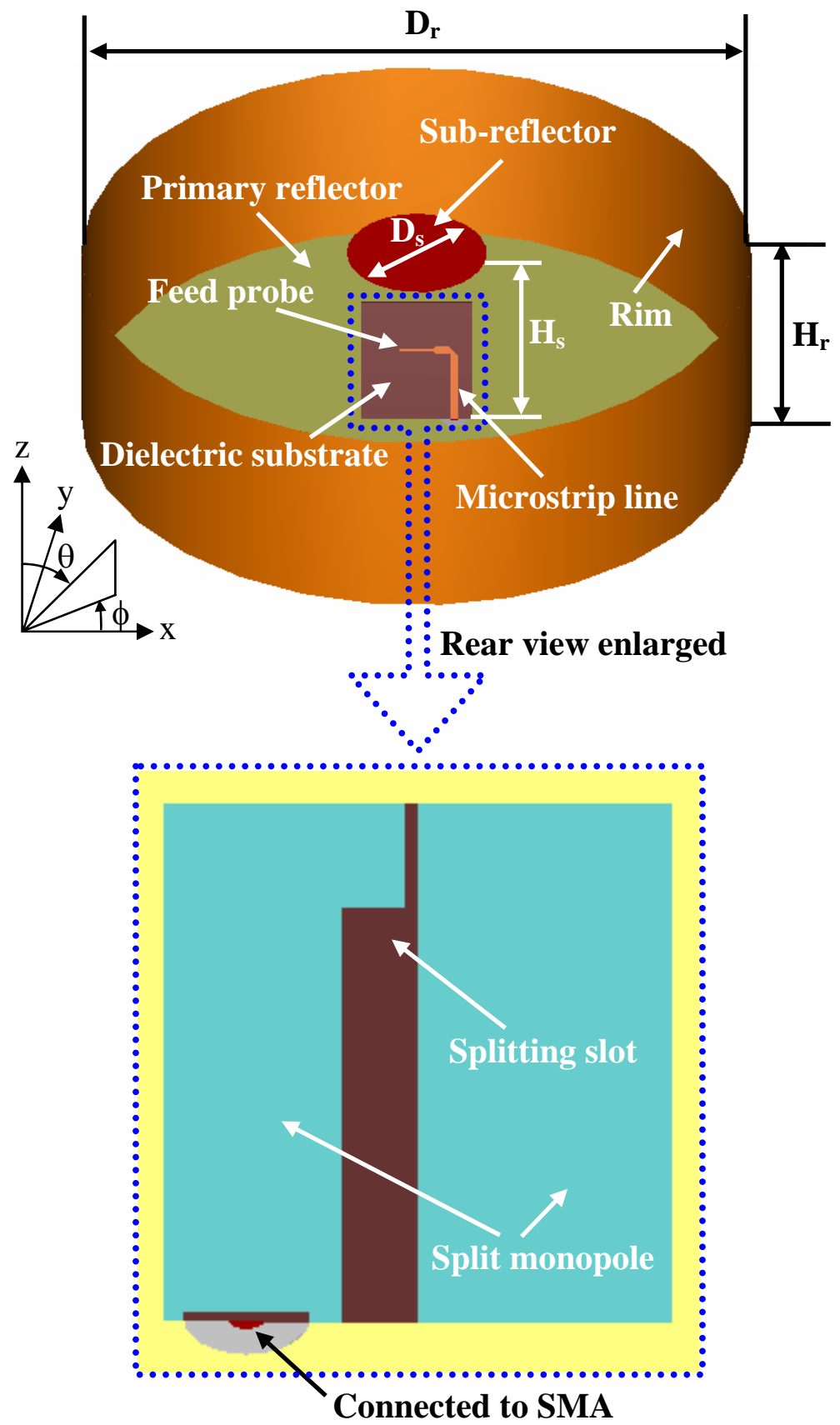

Fig. 1. Short backfire antenna excited by a new excitation structure which consists of a split monopole and a microstrip feed line (the dielectric cover on which the subreflector is printed is hidden). 


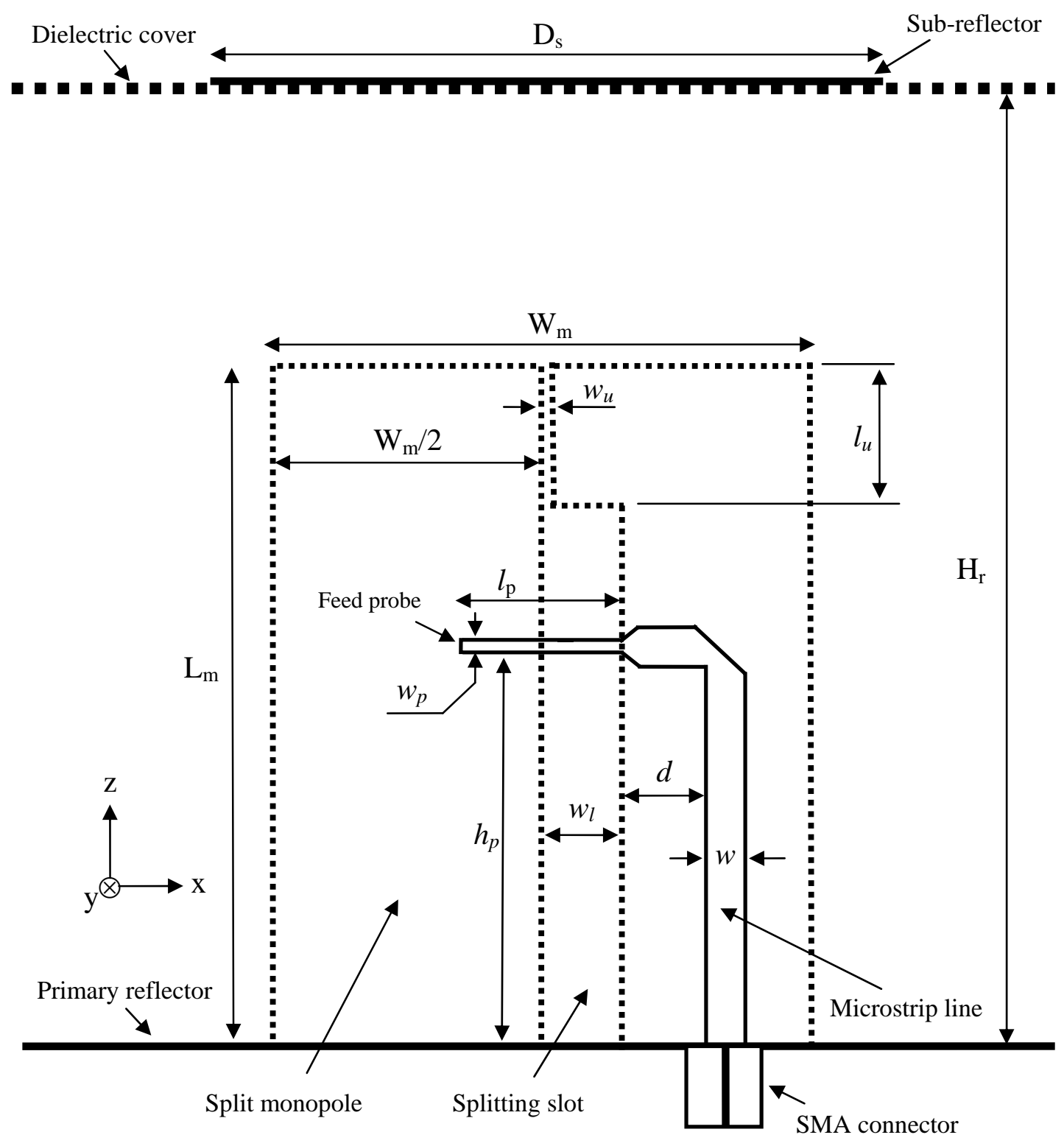

Fig. 2. A “close-up” view of the new excitation topology. 
Table I. Optimized geometric parameters for the split-monopole-excited SBA ( $\lambda_{0}=$ the free-space wavelength at the center frequency)

\begin{tabular}{|c|c|c|c|}
\hline $\mathrm{D}_{\mathrm{r}}$ & $120 \mathrm{~mm}\left(2.20 \lambda_{0}\right)$ & $w_{l}$ & $3 \mathrm{~mm}\left(0.055 \lambda_{0}\right)$ \\
\hline $\mathrm{H}_{\mathrm{r}}$ & $35 \mathrm{~mm}\left(0.64 \lambda_{0}\right)$ & $l_{p}$ & $6 \mathrm{~mm}\left(0.11 \lambda_{0}\right)$ \\
\hline $\mathrm{D}_{\mathrm{s}}$ & $25 \mathrm{~mm}\left(0.46 \lambda_{0}\right)$ & $h_{p}$ & $14.5 \mathrm{~mm}\left(0.266 \lambda_{0}\right)$ \\
\hline $\mathrm{L}_{\mathrm{m}}$ & $25 \mathrm{~mm}\left(0.46 \lambda_{0}\right)$ & $w_{p}$ & $0.5 \mathrm{~mm}\left(0.009 \lambda_{0}\right)$ \\
\hline $\mathrm{W}_{\mathrm{m}}$ & $20 \mathrm{~mm}\left(0.37 \lambda_{0}\right)$ & $d$ & $3 \mathrm{~mm}\left(0.055 \lambda_{0}\right)$ \\
\hline$l_{u}$ & $5 \mathrm{~mm}\left(0.09 \lambda_{0}\right)$ & $w$ & $1.5 \mathrm{~mm}$ \\
\hline$w_{u}$ & $0.5 \mathrm{~mm}\left(0.009 \lambda_{0}\right)$ & & \\
\hline
\end{tabular}




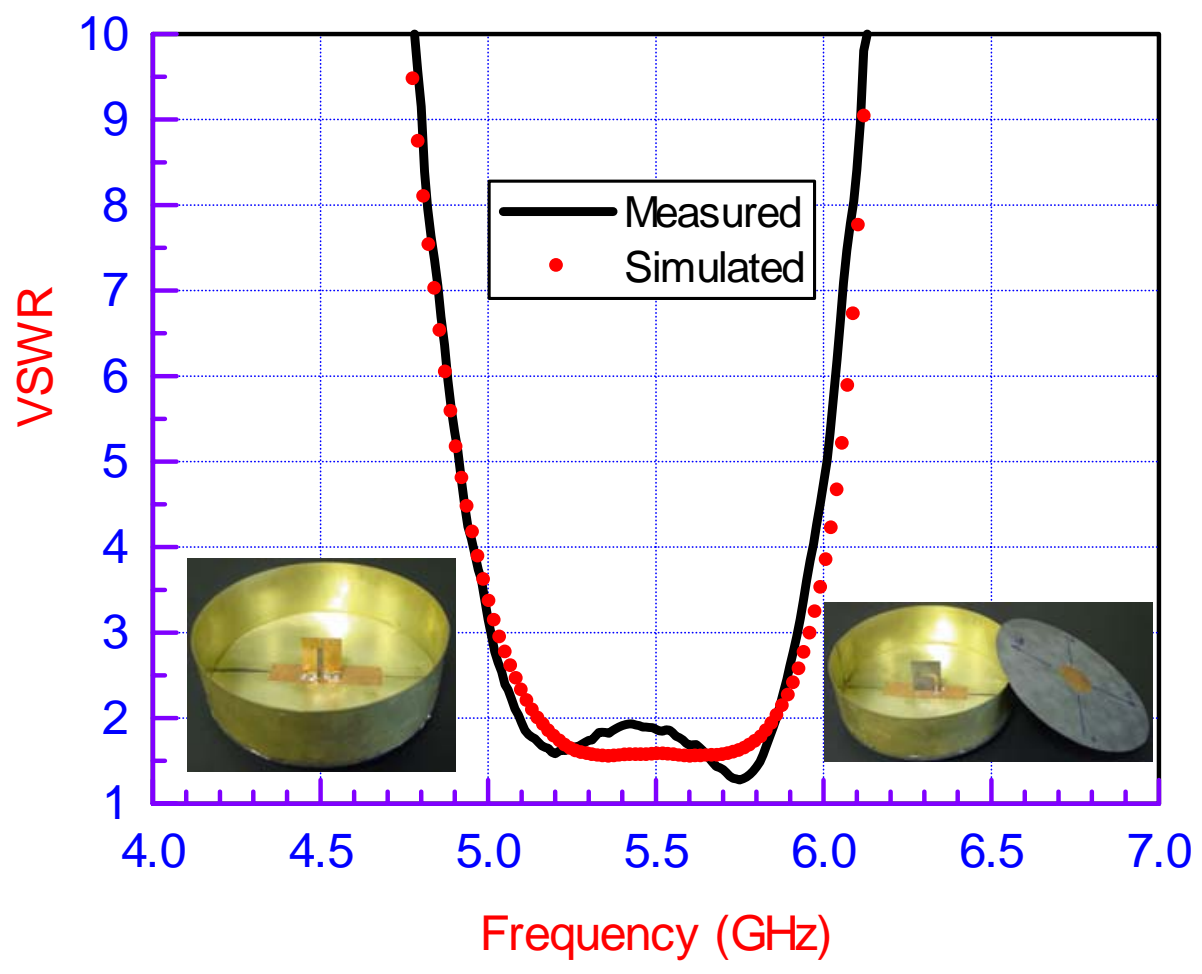

Fig. 3. Measured and simulated results of VSWR for the split-monopole-excited SBA (inset are two pictures of the antenna prototype). 


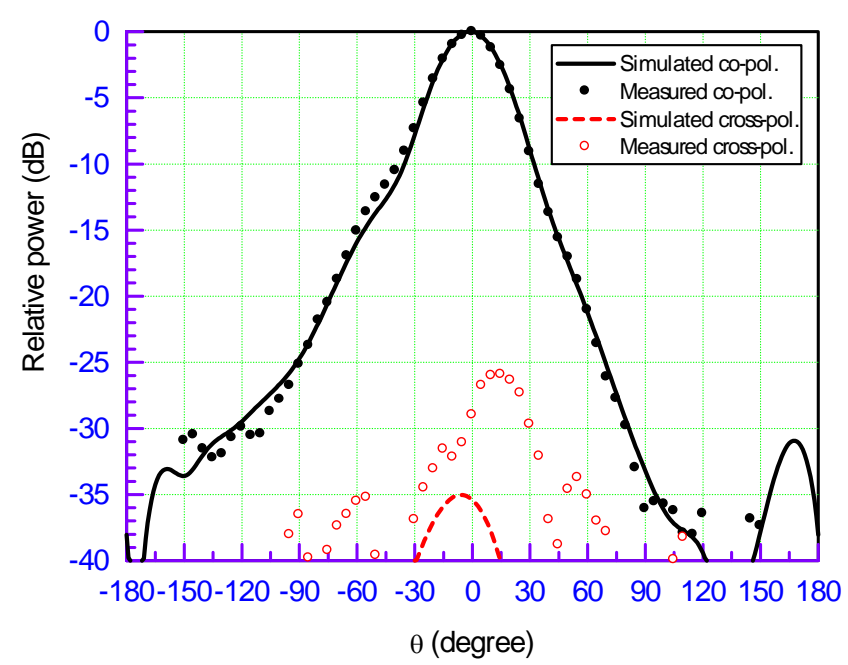

E-plane $\left(\phi=0^{\circ}\right)$

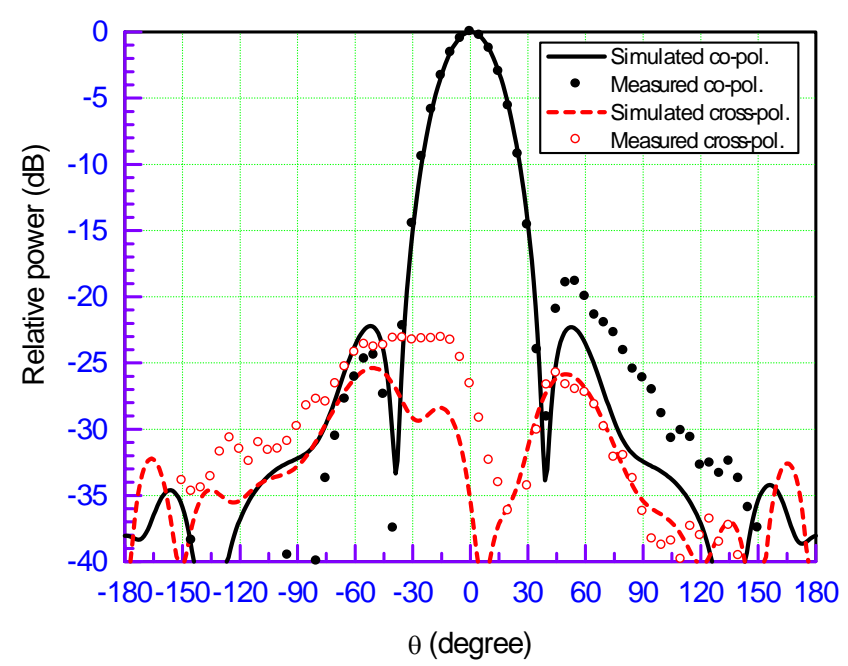

H-plane $\left(\phi=90^{\circ}\right)$

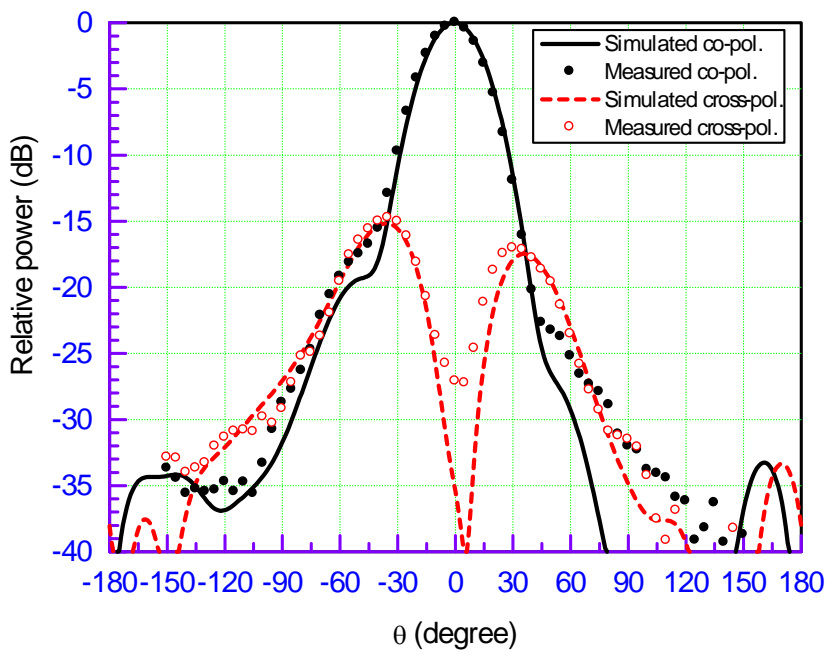

$\phi=45^{\circ}$ plane

(a) At $5.3 \mathrm{GHz}$

Fig. 4. Comparison of measured and simulated radiation patterns. 


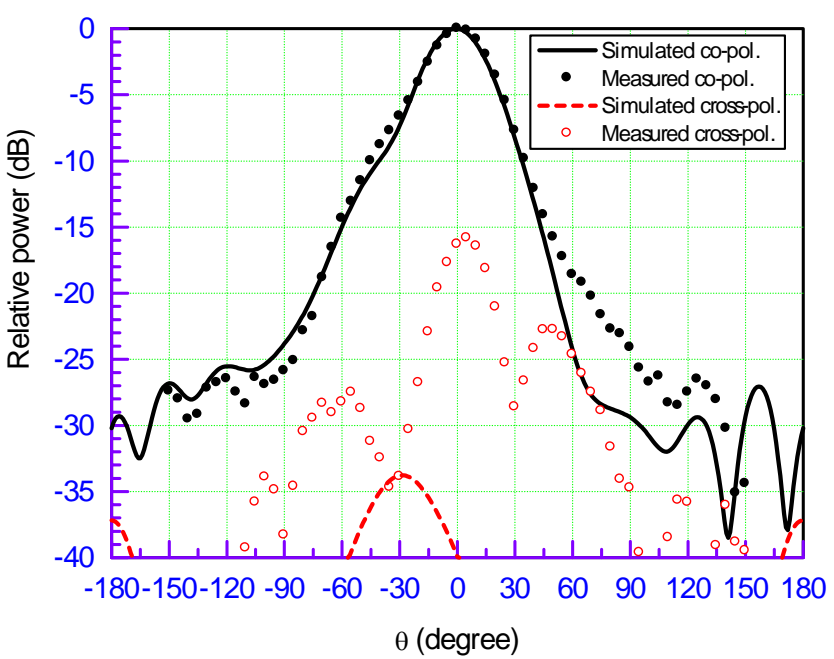

E-plane $\left(\phi=0^{\circ}\right)$

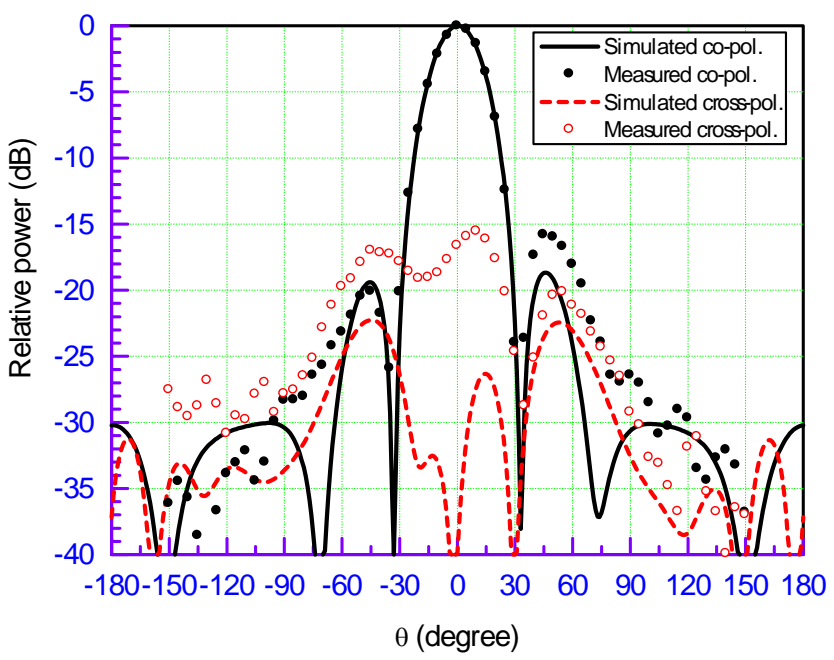

H-plane $\left(\phi=90^{\circ}\right)$

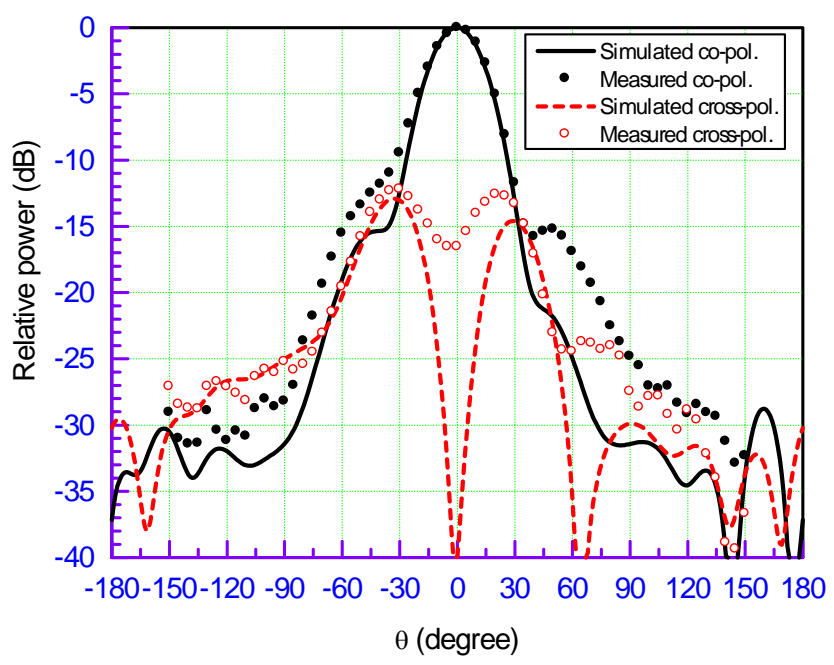

$\phi=45^{\circ}$ plane

(b) At $5.7 \mathrm{GHz}$ 


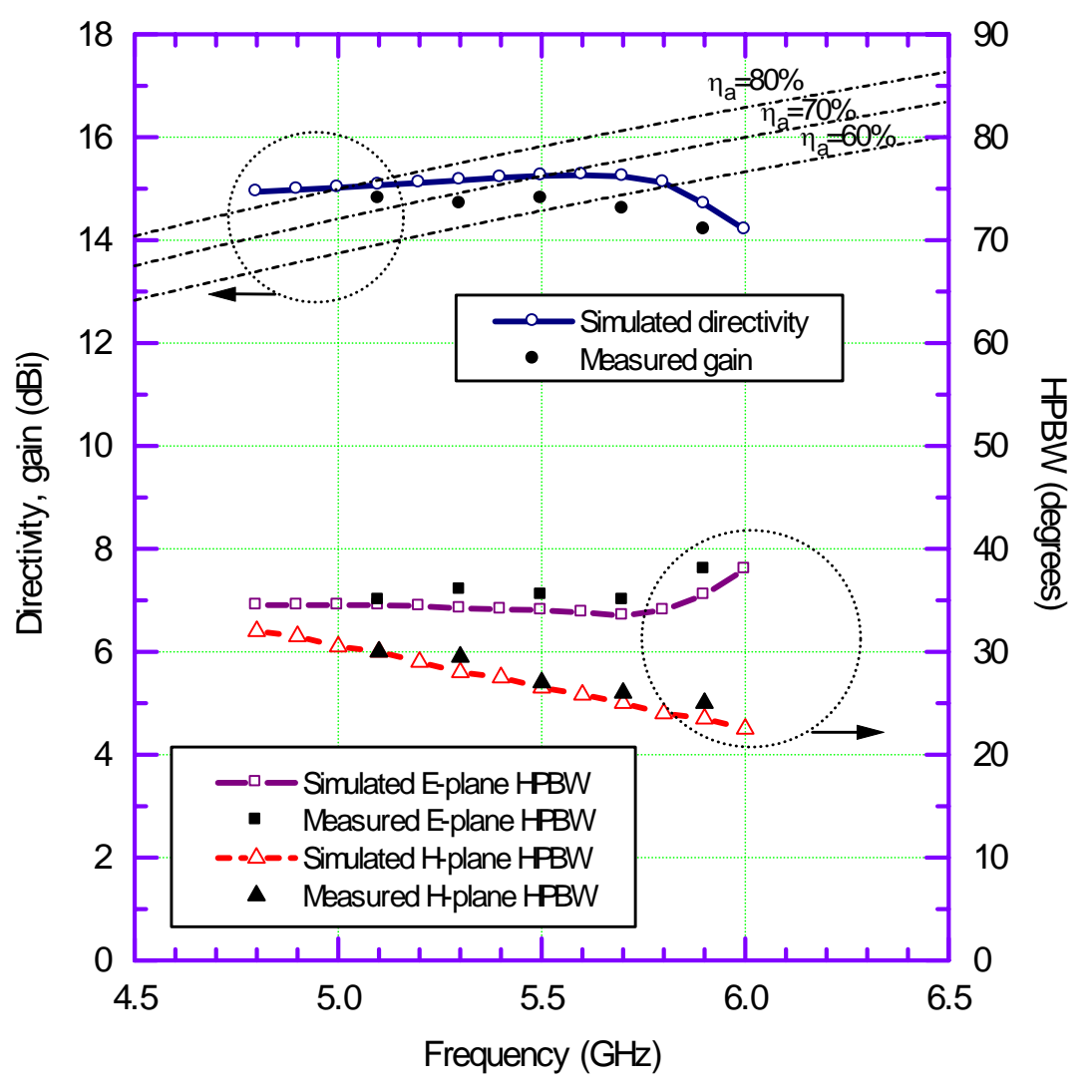

Fig. 5. Directivity, gain, and half-power beam width (HPBW) as a function of frequency. 


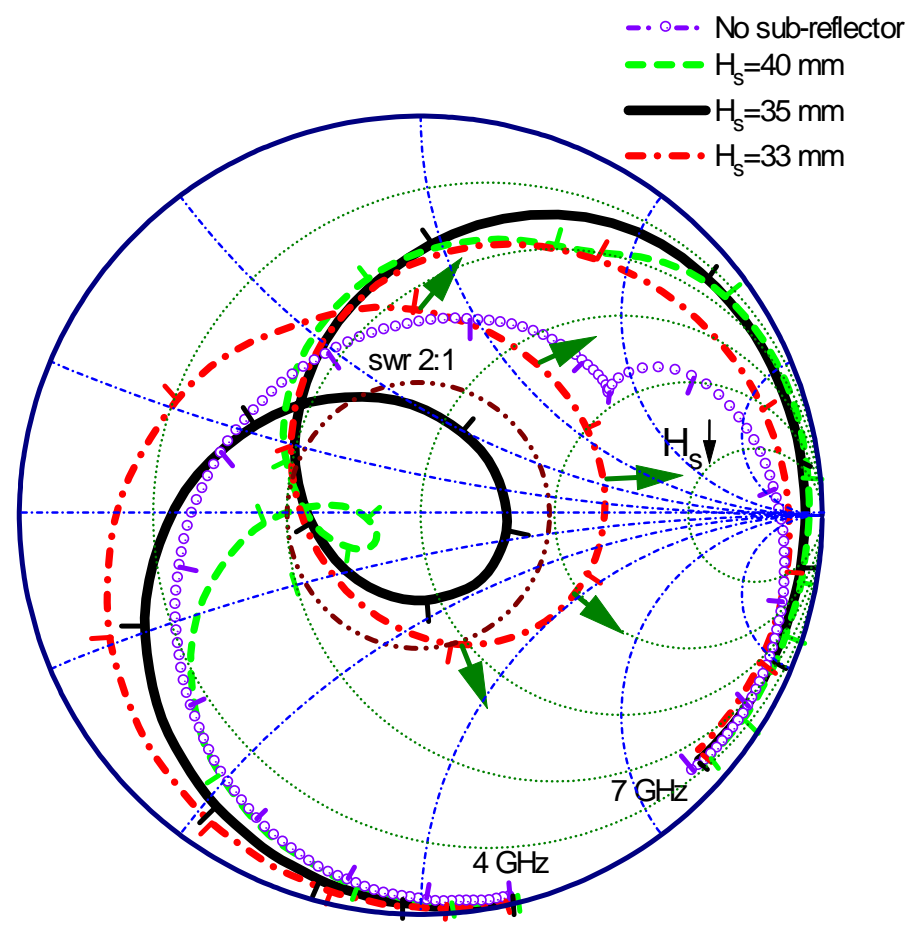

Fig. 6. Effect of the sub-reflector on the impedance performance. 


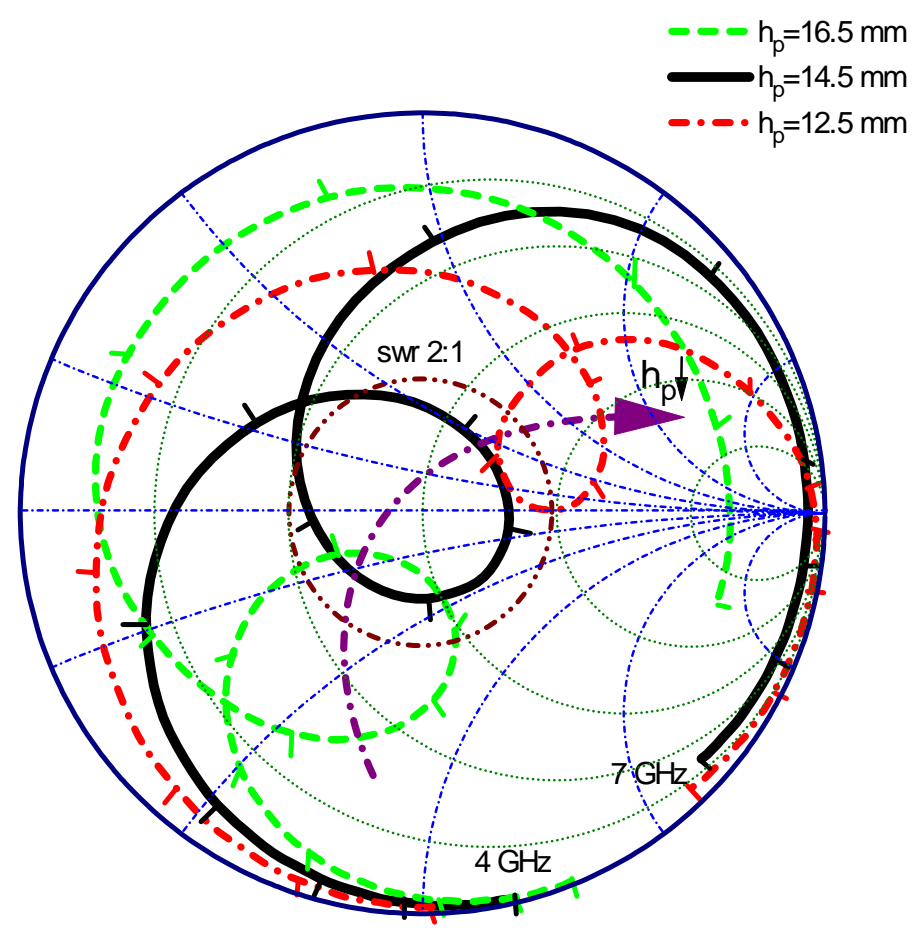

(a) Effect of the height of the feed probe $\left(h_{p}\right)$

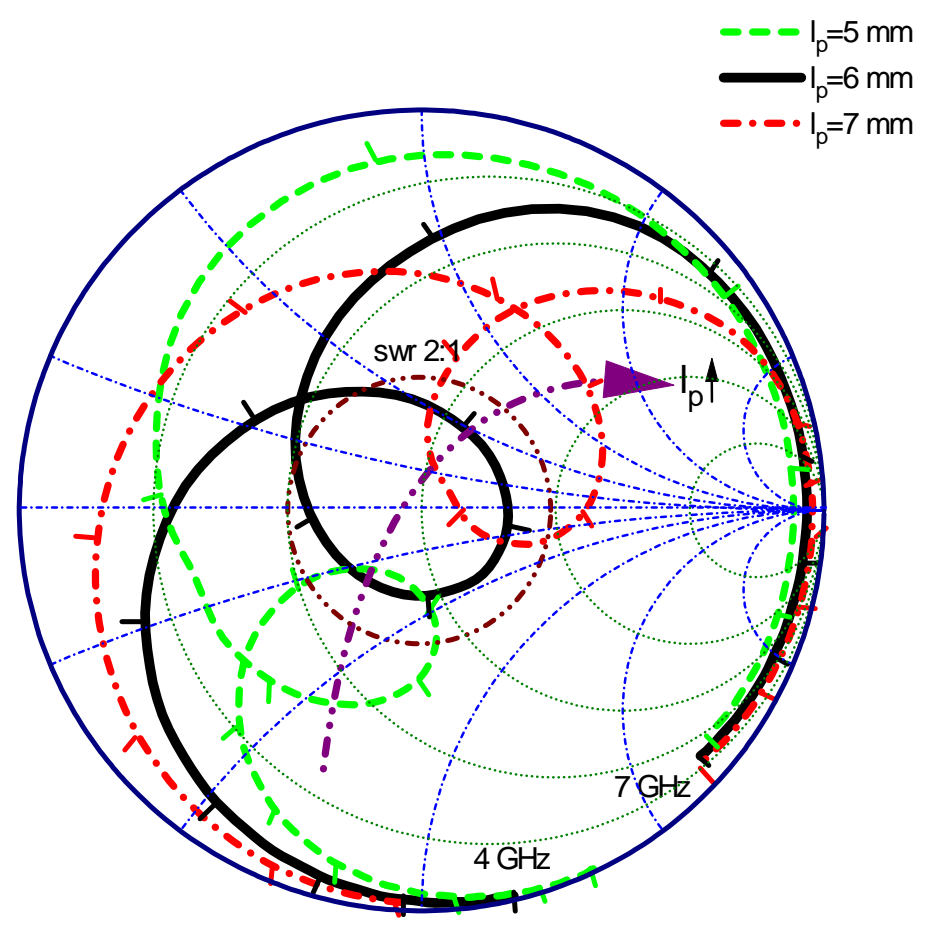

(b) Effect of the length of the feed probe $\left(l_{p}\right)$

Fig. 7. Effect of the feed probe on the impedance performance. 


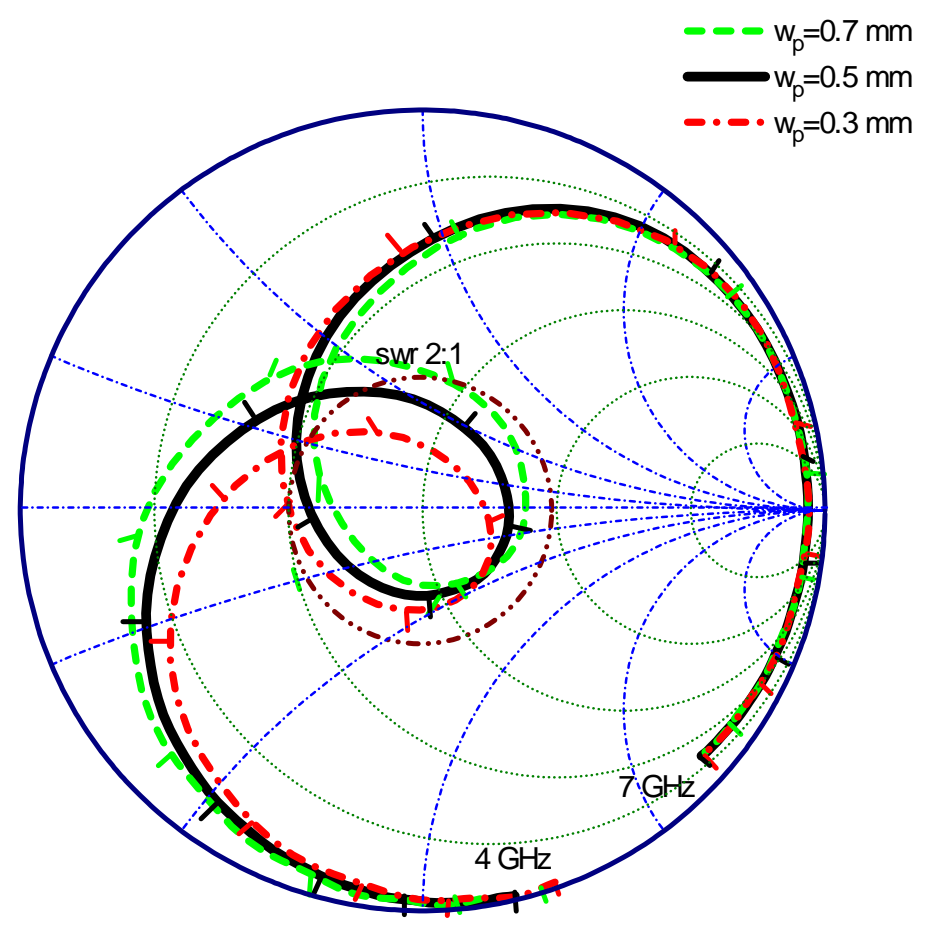

(c) Effect of the width of the feed probe $\left(\mathrm{w}_{\mathrm{p}}\right)$ 


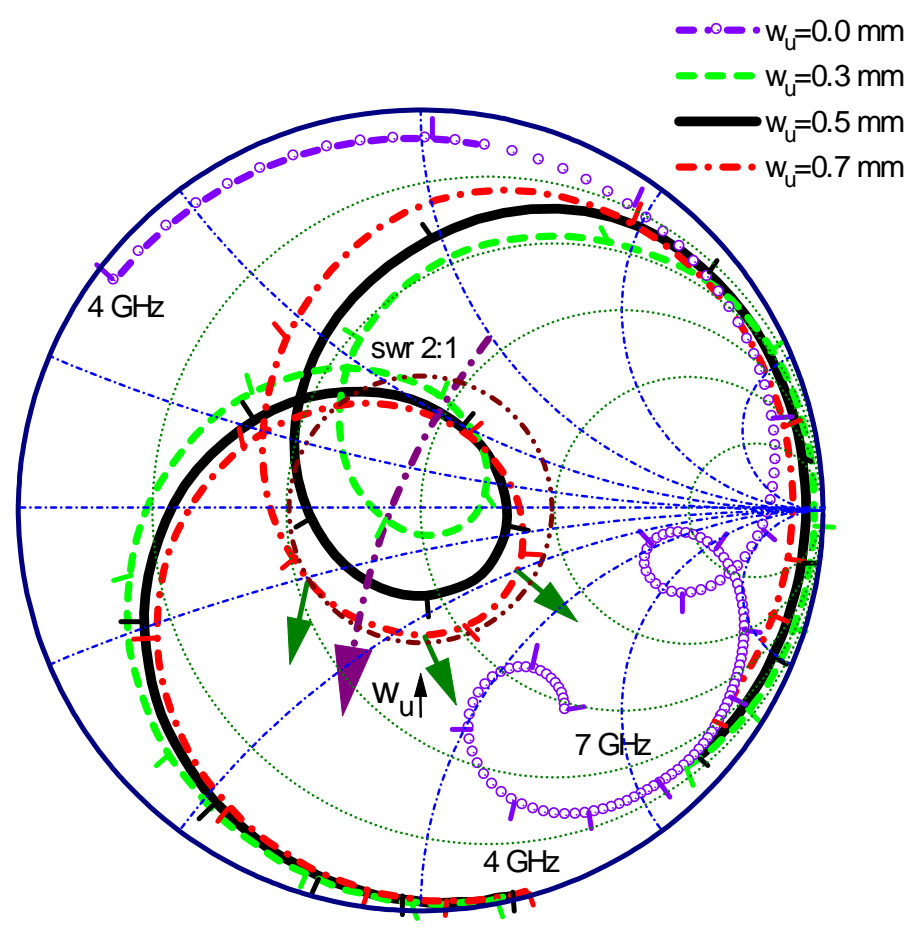

(a) Effect of the width of the upper rectangular slot $\left(w_{\mathrm{u}}\right)$

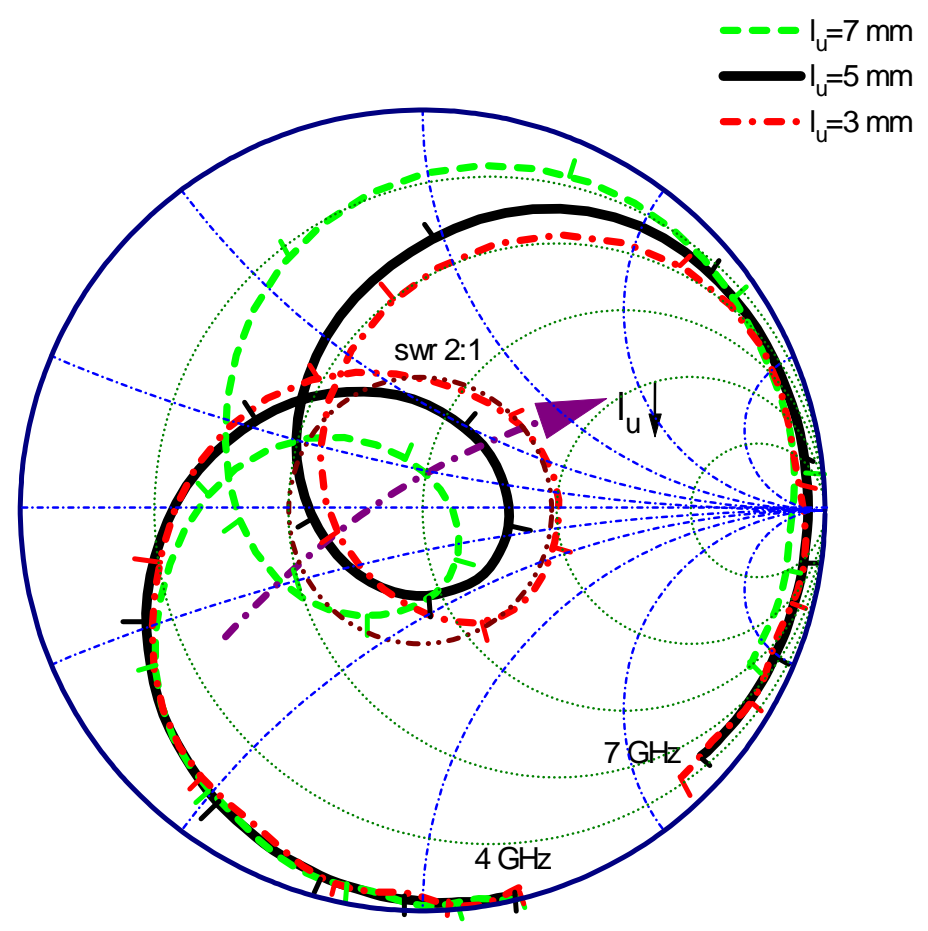

(b) Effect of the length of the upper rectangular slot $\left(l_{u}\right)$

Fig. 8. Effect of the splitting slot on the impedance performance. 


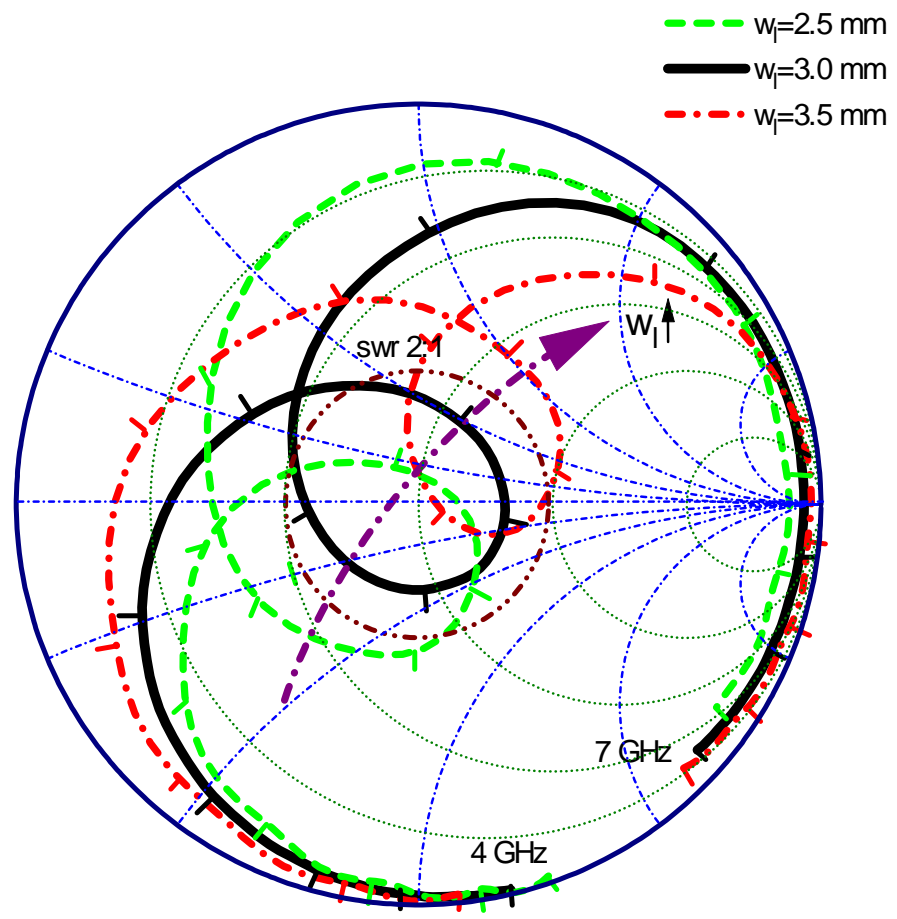

(c) Effect of the width of the lower rectangular slot $\left(w_{l}\right)$ 


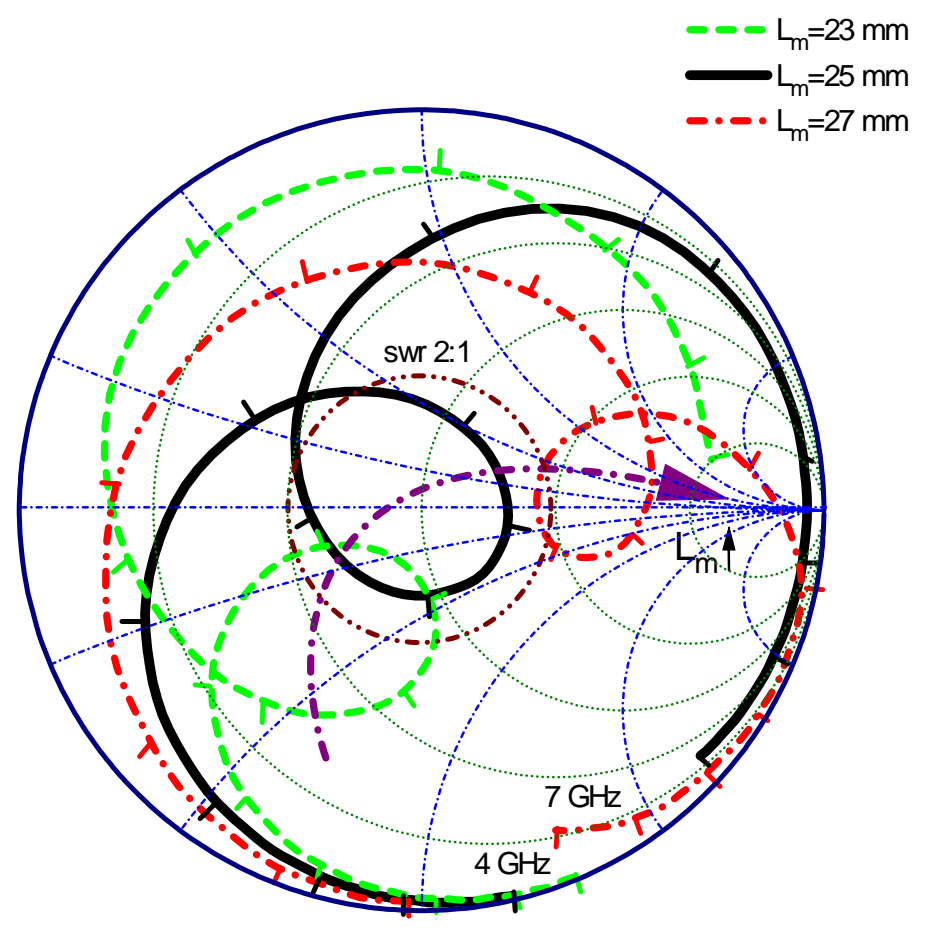

(a) Effect of the length of the split monopole $\left(\mathrm{L}_{\mathrm{m}}\right)$

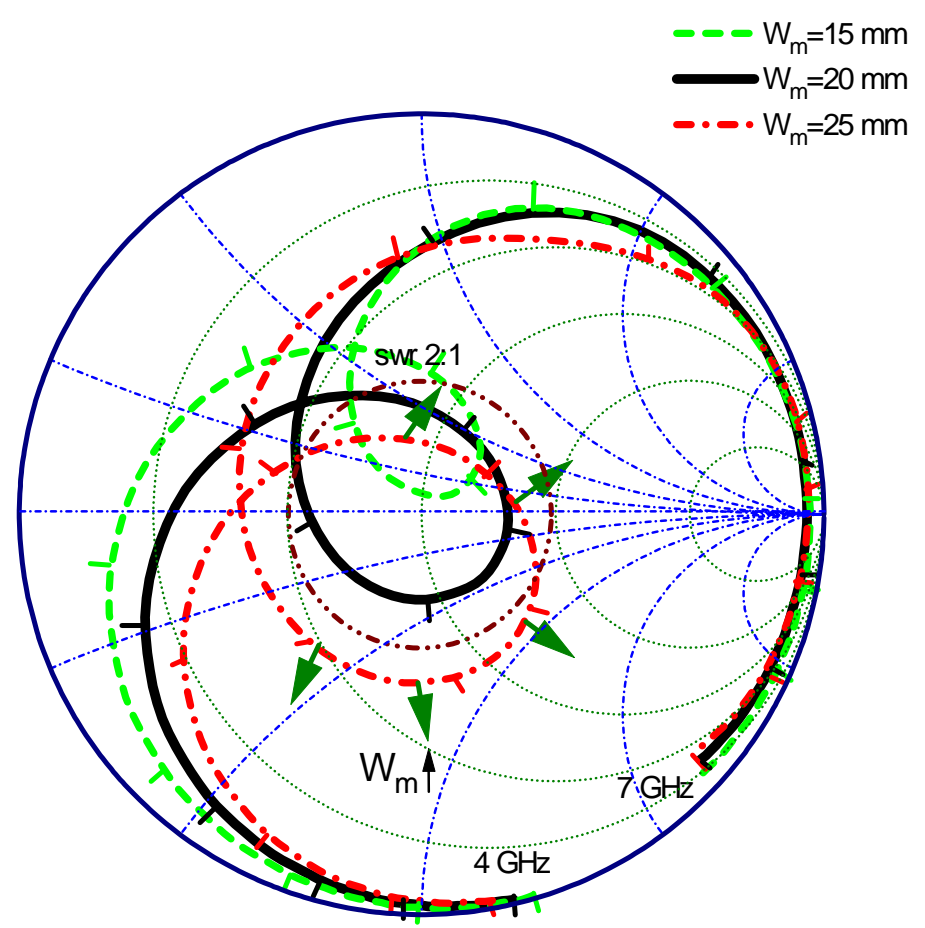

(b) Effect of the width of the split monopole $\left(\mathrm{W}_{\mathrm{m}}\right)$

Fig. 9. Effect of the split monopole on the impedance performance. 